九州大学学術情報リポジトリ

Kyushu University Institutional Repository

Insulin-like growth factor II messenger RNAbinding protein-3は乳腺悪性葉状腫瘍の指標になる

瀧澤，克実

https://doi. org/10.15017/1928638

出版情報：Kyushu University，2017，博士（医学），論文博士 バージョン：

権利関係: ๑ 2016 Elsevier Inc. All rights reserved. 


\title{
Insulin-like growth factor II messenger RNA- binding protein-3 is an indicator of malignant phyllodes tumor of the breast ${ }^{2 / 3}$
}

\author{
Katsumi Takizawa MD ${ }^{a}$, Hidetaka Yamamoto MD, PhD ${ }^{a}$, Kenichi Taguchi MD, PhD ${ }^{b}$, \\ Shinji Ohno MD, PhD ${ }^{c, d}$, Eriko Tokunaga MD, PhD ${ }^{c, e}$, Nami Yamashita MD, PhD ${ }^{\mathrm{e}}$, \\ Makoto Kubo MD, PhD ${ }^{f}$, Masafumi Nakamura MD, PhD ${ }^{f}$, Yoshinao Oda MD, PhD ${ }^{a, *}$
}

${ }^{a}$ Department of Anatomic Pathology, Graduate School of Medical Sciences, Kyushu University, Fukuoka, 812-8582, Japan

${ }^{\mathrm{b}}$ Department of Pathology, National Kyushu Cancer Center, Fukuoka, 811-1395, Japan

${ }^{\mathrm{c}}$ Department of Breast Oncology, National Kyushu Cancer Center, Fukuoka, 811-1395, Japan

${ }^{\mathrm{d}}$ Department of Breast Oncology, Cancer Institute Hospital, Tokyo, 135-8550, Japan

${ }^{\mathrm{e} D e p a r t m e n t ~ o f ~ S u r g e r y ~ a n d ~ S c i e n c e, ~ G r a d u a t e ~ S c h o o l ~ o f ~ M e d i c a l ~ S c i e n c e s, ~ K y u s h u ~ U n i v e r s i t y, ~ F u k u o k a, ~ 811-8582, ~ J a p a n ~}$

${ }^{\mathrm{f}}$ Department of Surgery and Oncology, Graduate School of Medical Sciences, Kyushu University, Fukuoka, 811-8582, Japan

Received 15 December 2015; revised 30 March 2016; accepted 13 April 2016

\author{
Keywords: \\ Phyllodes tumor; \\ IMP3; \\ EGFR; \\ Immunohistochemistry; \\ Prognosis
}

\begin{abstract}
Summary The aim of this study was to elucidate the clinicopathological and prognostic significance of the expressions of insulin-like growth factor II mRNA-binding protein-3 (IMP3) and epidermal growth factor receptor (EGFR) in phyllodes tumors (PTs). Immunohistochemical staining for IMP3 and EGFR was performed in 130 cases of primary PTs ( 83 benign, 28 borderline, 19 malignant), 34 recurrent/metastatic PTs, and 26 fibroadenomas (FAs). Among the primary tumors, a high expression of IMP3 was significantly more frequently present in malignant PTs $(17 / 19,89 \%)$ than in the FAs $(0 / 26,0 \%)$, benign PTs $(0 / 83,0 \%)$ and borderline PTs $(3 / 28,11 \%)$. The recurrent and metastatic lesions of malignant PTs also showed high IMP3 expression (3/5 [60\%] and 6/6 [100\%], respectively). Most malignant PTs showed strong IMP3 expression at the interductal area or more diffusely, whereas weak and focal (low) expression of IMP3 was limited to the periductal area in FAs and benign PTs. EGFR overexpression was significantly correlated with tumor grade and high IMP3 expression. Overexpressions of IMP3 and EGFR were significantly associated with shorter periods of metastasis-free and disease-free survival. The results suggest that high expressions of IMP3 and EGFR with a characteristic staining pattern may be helpful for both identifying malignant PT and predicting the prognosis of these tumors.

(C) 2016 Elsevier Inc. All rights reserved.
\end{abstract}

\footnotetext{
Competing interests: All authors have no conflict of interest related to this study. There is no funding support to disclose.

* Corresponding author at: Department of Anatomic Pathology, Pathological Sciences, Graduate School of Medical Sciences, Kyushu University, 3-1-1 Maidashi, Higashi-ku, Fukuoka 812-8582, Japan.

E-mail address: oda@surgpath.med.kyushu-u.ac.jp (K. Takizawa).
}

\section{Introduction}

Phyllodes tumor (PT) is an uncommon fibroepithelial neoplasm of the breast; it accounts for $<1 \%$ of all breast lesions [1]. PTs are histologically classified into benign, borderline and malignant categories on the basis of a combination of 
histological features, including the degree of stromal hypercellularity, mitoses and cytological atypia, stromal overgrowth, and the nature of the tumor borders/margins [2]. The frequencies of local recurrence and metastasis generally correlate with the histological grade of PT, but PT is a prognostically unpredictable neoplasm. The average in published data suggests an overall $21 \%$ local recurrence rate, including $17 \%, 25 \%$ and $27 \%$ recurrence rates for benign, borderline and malignant PTs, respectively. In addition, up to $10 \%$ of PTs seemingly show distant metastases, with $0 \%, 4 \%$ and $22 \%$ rates for benign, borderline and malignant PTs, respectively [3]. Local recurrence after surgery is also strongly influenced by the width of the excision margins [4]. To date, various markers such as p53, Ki-67 and c-kit have been reported to be overexpressed in stromal cells as a potential predictor of aggressive behavior of PT (reviewed by Tse et al [1] and Jara-Lazaro and Tan [5]). On the other hand, epithelial expression of estrogen receptor reportedly decreased along with increasing tumor grade [6]. However, the molecular mechanisms underlying the progression of PTs have not been fully clarified.

Insulin-like growth factor II (IGF-II) messenger RNA (mRNA)-binding protein-3 (IMP3), which has also been referred to as $\mathrm{K}$ homology domain containing-protein and as L523S, is a member of the IMP family composed of IMP1, IMP2, and IMP3 [7]. IMP3 binds to the $5^{\prime}$ untranslated region of the IGF-II leader-3 mRNA as a translational activator of IGF-II leader-3 mRNA, which controls cell proliferation [8]. Various recent studies showed that IMP3 is both a useful diagnostic marker to distinguish malignancies from benign lesions, and a prognostic marker for worse prognosis in several types of neoplasms, including mesenchymal tumors [9-11]. Regarding breast tumors, an experimental study of triple-negative breast cancer (TNBC) revealed that epidermal growth factor receptor (EGFR) signaling and the consequent activation of the mitogen-activated protein kinase pathway induced IMP3 transcription and protein expression, and IMP3 contributed to the migration and invasion of carcinoma cells [12]. As for PT, previous studies showed that EGFR expression was higher in advanced-grade PTs, suggesting that EGFR overexpression may be involved in the progression of PTs $[13,14]$. Thus, we hypothesized that IMP3 and EGFR might become biomarkers of malignant PT. A very recent study on a total of 62 cases of PTs has shown that IMP3 was expressed more frequently in malignant PTs than in borderline or benign PTs [15]. However, the prognostic value of IMP3 overexpression and its association with EGFR in PTs have not been fully elucidated so far.

In the present study, we examined a large series of PT to elucidate the diagnostic and prognostic significances of IMP3 and EGFR.

\section{Materials and methods}

We reviewed the cases of PTs that had been diagnosed at the Department of Anatomic Pathology of Kyushu University and the Department of Pathology of the National Kyushu Cancer Center between 1983 and 2014. Our study protocol conformed to the ethical guidelines of the 1975 Declaration of Helsinki. For strict privacy protection, the identifying information for all samples was removed before our analyses. This study was approved by the institutional review boards of Kyushu University (No. 26-186) and the National Kyushu Cancer Center (No. 2015-8).

We obtained a total of 164 cases of surgically resected PTs. The examined PTs were primary $(\mathrm{n}=130)$, recurrent $(\mathrm{n}=28)$ and metastatic $(n=6)$ tumors. PTs were classified into benign (primary, $\mathrm{n}=83$; recurrent, $\mathrm{n}=18$ ), borderline (primary, $\mathrm{n}=$ 28 ; recurrent, $\mathrm{n}=5$ ) and malignant (primary, $\mathrm{n}=19$; recurrent, $\mathrm{n}=5$; metastasis, $\mathrm{n}=6$ ) on the basis of histological features according to the World Health Organization (WHO) classification (see below) [2]. Follow-up information was obtained by reviewing the medical records of the patients and communicating with the physicians at each facility. In this series, metastasis occurred in 10 cases (see Results). Among these 10 cases, histological specimens of metastatic foci were available in six cases. As controls, 26 cases of primary fibroadenoma (intracanalicular type, $\mathrm{n}=14$; pericanalicular type, $\mathrm{n}=10$; juvenile type, $\mathrm{n}=2$ ) were also used.

\subsection{Pathological review}

All cases were reviewed by two pathologists (K.T., H.Y.). Tumors were scored for stromal cellularity (score 1-3) and stromal nuclear atypia (score 1-3) (Fig. 1A, D, G). We defined stromal cellularity as follows; score 1 , if the cellularity was low (stromal cells were sparsely distributed, but the cellularity was higher than that of fibroadenoma); score 2, if the cellularity was moderate (ie, between low and high), and score 3 , if the cellularity was high (stromal cells showed a back-to-back arrangement). When the cellularity was heterogeneous within an individual tumor, the higher score was recorded. Slightly increased cellularity only at the periductal area was acceptable for "low" cellularity.

Stromal nuclear atypia was judged as score 1 if the cells were slender spindle cells, but the nuclei were slightly larger than that of fibroadenoma; score 2, if the cells were relatively uniform spindle cells with more plump nuclei than that of score 1 , and the cells were morphologically similar to the spindle cells present in low-grade malignant fibroblastic/myofibroblastic spindle cell tumors of soft tissue such as desmoid tumor or adult fibrosarcoma; score 3, if the stromal cells showed frankly malignant or sarcomatous appearance with nuclear pleomorphism, morphologically identical to undifferentiated pleomorphic sarcoma of soft tissue (Fig. 1A, D, G).

The stromal mitotic activity was quantified per 10 highpower fields (HPFs) in the most mitotically active areas of the stroma. Heterologous differentiation components such as rhabdomyosarcoma, chondrosarcoma and osteosarcoma were screened by hematoxylin-eosin staining and confirmed by additional immunohistochemical stains such as myogenin, 

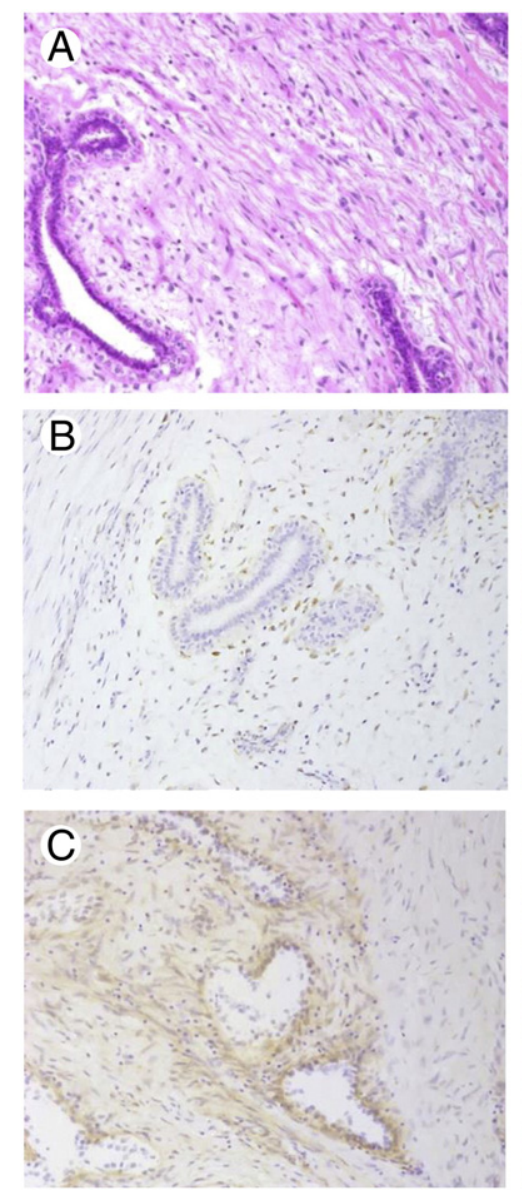
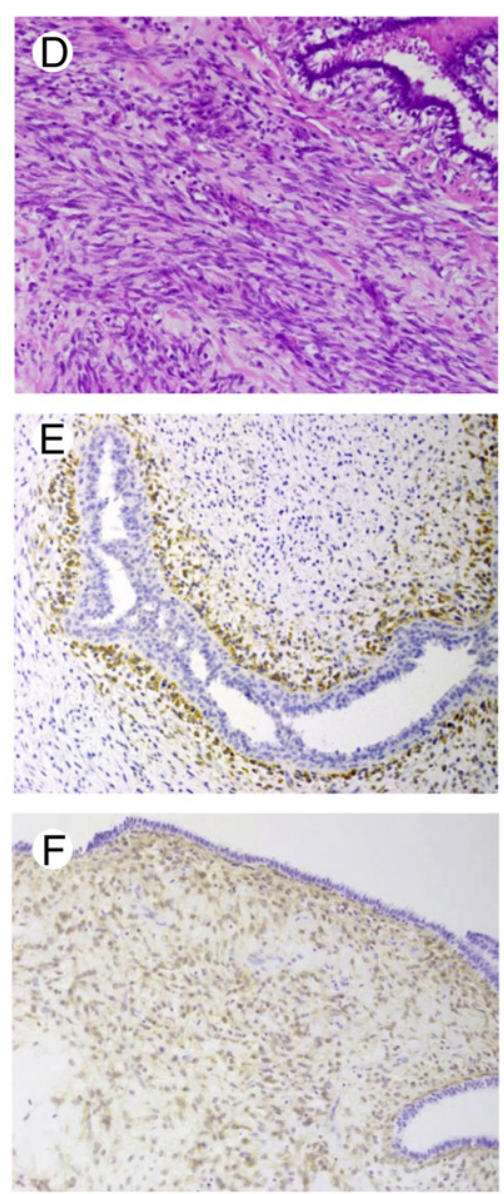
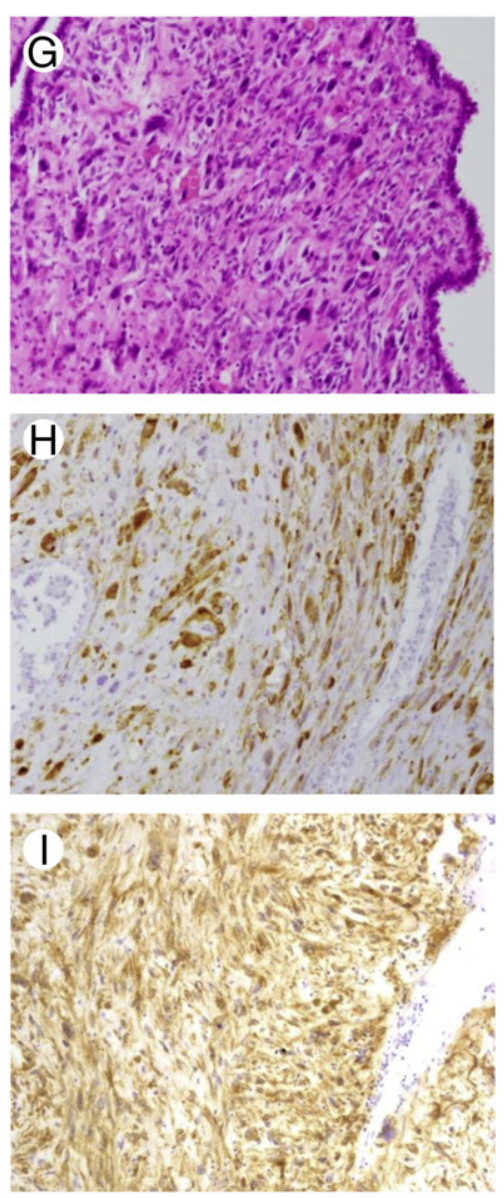

Fig. 1 Histological and immunohistochemistry (IHC) findings in PTs (benign, A-C, borderline, D-F; malignant, G-I) (hematoxylin-eosin stain, A, D, G, IMP3, B, E, H, EGFR, C, F, I) (original magnification $\times 200$ ). A, Benign PT shows a low stromal cellularity (stromal cellularity score 1) and slender spindle-shaped nuclei (nuclear atypia score 1). B, IHC for IMP3 shows focal and weak expression only at the periductal area, judged as low expression. C, EGFR expression with moderate intensity is observed only at the periductal area, judged as positive. D, Borderline PT shows a moderate stromal cellularity (stromal cellularity score 2) and spindle-shaped plumped nuclei (nuclear atypia score 2). E, IHC for IMP3 shows focal and strong expression only at the periductal area, judged as high expression. F, EGFR expression with moderate intensity is present at the periductal and interductal area, judged as positive. G, Malignant PT shows high stromal cellularity (stromal cellularity score 3) and marked stromal nuclear pleomorphism (nuclear atypia score 3). H and I, IHC for IMP3 and EGFR shows diffuse and strong expression, judged as high expression/positive.

desmin and S-100 protein. Surgical margins were not included in the histological evaluation, because the margin information was not available from the medical records in several cases.

Histological grade of PT was determined as follows: basically, we classified PTs into benign, borderline and malignant, according to the low (score 1), moderate (score 2) and high (score 3) grade nuclear atypia, respectively. In addition, tumor grade was modified by cellularity and/or mitotic counts. For example, PT with moderate atypia in the very limited area was defined as benign, if cellularity was entirely low. PT with low-grade atypia and entirely high cellularity was defined as borderline. Moderately atypical cases with both $\geq 10$ mitoses/10 HPFs and diffusely high cellularity were upgraded to malignant. Moderately atypical cases with either $\geq 10$ mitoses/10 HPFs or diffusely high cellularity were classified into borderline. Malignant, borderline and benign PTs basically showed $\geq 10$, $\leq 9$ and $\leq 4$ mitoses per $10 \mathrm{HPFs}$, respectively, with a few exceptions. We didn't apply tumor size for definition of tumor grade, like WHO classification.

\subsection{Immunohistochemical staining and evaluation}

Immunohistochemical staining was performed on $4-\mu \mathrm{m}$ thick formalin-fixed, paraffin-embedded tissue sections. Immunohistochemical expression of each marker was evaluated in the stromal component of PT. As for IMP3, antigen retrieval was achieved by heating the sections in a microwave for $20 \mathrm{~min}$ in a target retrieval solution (Dako, Carpinteria, CA, USA). The slides were incubated with mouse monoclonal antibody against IMP3 (clone 49.1; dilution $\times 100$; Dako) for $1 \mathrm{~h}$. at room temperature and then reacted with the Envision Plus system (Dako) for $30 \mathrm{~min}$ at room temperature. The labeled antigens were visualized by $3,3^{\prime}$-diaminobenzidine tetrahydrochloride as a chromogen and finally counterstained 
with hematoxylin. We used the germinal center of the lymphoid follicles for an external positive control for IMP3, as described [16].

We evaluated the degree of immunohistochemical expression of IMP3 according to our previous study [10]; the staining intensity for IMP3 was considered strong if cytoplasmic staining was easily observed at low-power magnification, weak if there was fuzzy staining that could not be easily observed at low-power magnification, and absent if there was no staining at all. The proportion of immunoreactive cells for IMP3 was classified as diffuse ( $\geq 50 \%$ ) or focal ( $1 \%-49 \%$ ). Cases with diffuse or focal expression and strong staining intensity for IMP3 and cases with diffuse expression and weak staining intensity were judged as high-expression cases, whereas those with focal expression and weak staining intensity for IMP3 or no staining for IMP3 were judged as lowexpression. Regarding the intratumoral localization, IMP3 expression could be classified as periductal or interductal pattern (see Results). Periductal pattern was that stromal cells only adjacent to the ductal/epithelial element expressed IMP3, whereas interductal pattern was that stromal cells between the ducts expressed IMP3.

For immunohistochemical staining for EGFR, we used a mouse monoclonal antibody for EGFR ( $31 \mathrm{G} 7$; dilution $\times 10$; Zymed, South San Francisco, CA). For the evaluation of EGFR expression, membranous and/or cytoplasmic staining of tumor cells was regarded as a consistent staining pattern. The staining intensity was classified into weak, moderate or strong, and immunoreactivity for EGFR with a moderate to strong staining intensity in $\geq 10 \%$ of tumor cells was judged as positive, as described previously [13].

Immunohistochemical staining for Ki-67 (mouse monoclonal, MIB-1, Dako), myogenin (mouse monoclonal, F5D, Dako), $\alpha$-smooth muscle actin (mouse monoclonal, 1 A4, Sigma BioScience, St. Louis, MO), desmin (mouse monoclonal, D33, Dako) and S-100 protein (rabbit polyclonal, Dako) was performed to confirm the heterologous malignant component.

\subsection{Statistical analyses}

Statistical analyses were performed by Fisher's exact test. Survival curves were calculated using the Kaplan-Meier method, with significance assessed by the log-rank test. We estimated disease-free survival (DFS), which was defined as the length of time from the date of surgery to the date of a first event (such as local recurrence or metastasis) or to the last follow-up date for censored cases. Metastasis-free survival (MFS) was defined as the length of time from the date of surgery to the date of the first distant metastasis of PT or to the last follow-up date for censored cases.

Overall survival (OS) was defined as the length of time from the date of surgery to death related to PT or to the last follow-up date for censored cases. We used univariate and multivariate regression analyses with Cox proportional hazard models. All calculations were performed using
JMP software ver. 11 (SAS Institute, Cary, NC). $P<.05$ was considered significant.

\section{Results}

\subsection{Clinicopathological findings}

The clinicopathological findings of the 130 cases of primary PTs are summarized in Table 1. All of the patients were women, ranging in age from 11 to 77 years (median, 45 years old). The patients' tumor size, stromal cellularity, nuclear atypia, mitotic counts and Ki-67 labeling index were essentially parallel with the histological grades of their tumors; most of the malignant PTs showed larger tumor size (median, $9 \mathrm{~cm}$ ), high stromal cellularity (score 3), significant nuclear atypia (score 3 ), increased mitoses (>10/10 HPFs) and a higher Ki-67 labeling index (median, 20\%).

Heterologous malignant elements were found in five cases of malignant PT, including chondrosarcomatous component $(n=4)$, osteosarcomatous component $(n=3)$, rhabdomyosarcomatous component $(\mathrm{n}=2)$, and a combination of these components $(\mathrm{n}=3)$.

\subsection{IMP3 expression in each grade of PT}

The results of the immunohistochemistry for IMP3 are summarized in Table 2 and Supplementary Table 1. In the vast majority of fibroadenomas and benign PTs, IMP3 expression was absent, whereas only one of the 26 fibroadenoma cases (4\%) and five of the 83 benign PT cases (6\%) showed focal

Table 1 Clinicopathological characteristic of 130 cases of primary phyllodes tumors

\begin{tabular}{|c|c|c|c|c|}
\hline Factors & $\begin{array}{l}\text { Benign } \\
(\mathrm{n}=83)\end{array}$ & $\begin{array}{l}\text { Borderline } \\
(\mathrm{n}=28)\end{array}$ & $\begin{array}{l}\text { Malignant } \\
(\mathrm{n}=19)\end{array}$ & $\begin{array}{l}\text { Total } \\
(\mathrm{n}=130)\end{array}$ \\
\hline Age (years) ${ }^{a}$ & $43(11-77)$ & $46(26-73)$ & $50(17-73)$ & $45(11-77)$ \\
\hline Size $(\mathrm{cm})^{\text {a }}$ & $4.7(1-13.5)$ & $7.2(2-18)$ & $9.0(3-20.5)$ & $5.2(1-20.5)$ \\
\hline \multicolumn{5}{|c|}{ Stromal cellularity } \\
\hline Score 1 & 73 & 0 & 0 & 73 \\
\hline Score 2 & 10 & 19 & 2 & 31 \\
\hline Score 3 & 0 & 9 & 17 & 26 \\
\hline \multicolumn{5}{|c|}{ Nuclear atypia } \\
\hline Score 1 & 78 & 3 & 0 & 81 \\
\hline Score 2 & 5 & 25 & 1 & 31 \\
\hline Score 3 & 0 & 0 & 18 & 18 \\
\hline \multicolumn{5}{|c|}{ Mitosis (/10 HPFs) } \\
\hline $0-4$ & 82 & 13 & 0 & 95 \\
\hline $5-9$ & 1 & 13 & 3 & 17 \\
\hline$\geq 10$ & 0 & 2 & 16 & 18 \\
\hline Ki-67 LI (\%) * & $2.0(0.2-14.2)$ & $9.5(1.3-28.2)$ & $20(5.9-79.8)$ & $3(0.2-79.8)$ \\
\hline
\end{tabular}


Table 2 IMP3 expression in fibroadenomas and each grade of phyllodes tumors

\begin{tabular}{ccccc}
\hline IMP3 expression & $\begin{array}{l}\text { Fibroadenoma } \\
(\mathrm{n}=26)\end{array}$ & $\begin{array}{l}\text { Benign PT } \\
(\mathrm{n}=83)\end{array}$ & $\begin{array}{l}\text { Borderline PT } \\
(\mathrm{n}=28)\end{array}$ & $\begin{array}{l}\text { Malignant PT } \\
(\mathrm{n}=19)\end{array}$ \\
\hline Low expression & $26(100 \%)$ & $83(100 \%)$ & $25(89 \%)$ & $2(11 \%)$ \\
No & $25(96 \%)$ & $78(94 \%)$ & $19(68 \%)$ & $2(11 \%)$ \\
Focal weak & $1(4 \%)$ & $5(6 \%)$ & $6(21 \%)$ & $0(0 \%)$ \\
High expression & $0(0 \%)$ & $0(0 \%)$ & $3(11 \%)$ & $17(89 \%)$ \\
Focal strong & $0(0 \%)$ & $0(0 \%)$ & $0(0 \%)$ & $6(31 \%)$ \\
Diffuse weak & $0(0 \%)$ & $0(0 \%)$ & $0(0 \%)$ & $0(0 \%)$ \\
Diffuse strong & $0(0 \%)$ & $0(0 \%)$ & & $11(58 \%)$ \\
\hline
\end{tabular}

Abbreviation: PT, phyllodes tumor.

weak expression of IMP3 only at the periductal area (Fig. 1B). Among the borderline PTs, IMP3 expression was absent (19/ 28 cases, $68 \%$ ), focal weak (6/28 cases, $21 \%$ ) and focal strong (3/28 cases, $11 \%)$ (Fig. 1E).

Regarding the localization of IMP3, five cases of borderline PT showed the periductal expression pattern (4 weak, 1 strong), three cases showed the interductal pattern (2 weak, 1 strong), and one case showed both interductal and periductal patterns with strong intensity. Among the malignant PTs, IMP3 expression was absent (2/19 cases, $11 \%)$, focal strong (6/19 cases, 31\%) and diffuse strong (11/19 cases, 58\%) (Fig. 1H). Seventeen cases of malignant PT showed a strong expression of IMP3, mainly at the interductal area with or without periductal area.

Collectively, among the primary PTs, a high expression of IMP3 was present in $0 \%$ ( $0 / 83$ cases) of the benign PTs, $11 \%$ ( $3 / 28$ cases) of the borderline PTs, and $89 \%$ (17/19 cases) of the malignant PTs (Fig. 2A). The high IMP3 expression was significantly more frequent in the higher-grade tumors (Fig. 2A). In addition, in 2 cases of malignant PT with heterogeneous components (benign and malignant components), high IMP3 expression was recognized in only the malignant component in the individual tumor. The heterologous sarcomatous elements observed in five cases of malignant PT also showed a high expression of IMP3.

Among the recurrent or metastatic tumors, $3(60 \%)$ of 5 cases of recurrent malignant PTs and all six cases (100\%) of metastatic malignant PT showed high IMP3 expression, whereas none of the recurrent lesions of benign and borderline PTs showed it (Fig. 2B).

The correlations between the IMP3 expression and clinicopathological parameters are summarized in Table 3 . The high expression of IMP3 was significantly correlated with larger tumor size $(>5 \mathrm{~cm}, P=.0064)$, higher stromal cellularity (scores 2 and 3, $P<.0001$ ) and stromal atypia (scores 2 and $3, P<.0001$ ), higher mitoses (5 or more/10 HPFs, $P<.0001)$ and higher Ki-67 labeling index $(>3 \%$, $P<.0001$ ), but not with the patients' age.

\subsection{EGFR expression}

EGFR was positive in $4 \%$ ( $1 / 26$ cases $)$ of fibroadenoma, $18 \%$ (15/83 cases) of benign PT, $54 \%$ (15/28 cases) of borderline PT, and $84 \%$ (16/19 cases) of malignant PT (Fig. 2C). The prevalence of EGFR positivity was significantly higher in the advanced-grade tumors (Fig. 2C). Among the recurrent or metastatic tumors, $3 / 18$ cases $(17 \%)$ of recurrent benign PT, $3 / 5$ cases $(60 \%)$ of recurrent borderline PT, $4 / 5$ cases $(80 \%)$ of recurrent malignant PT, and $5 / 6$ cases $(83 \%)$ of metastatic malignant PT were positive for EGFR (Fig. 2D).

EGFR overexpression was significantly correlated with a higher stromal cellularity score $(P<.0001)$ and a higher nuclear atypia score $(P<.0001)$, higher mitoses $(P<.0001)$ and higher Ki-67 labeling index $(P<.0001)$, but it was not correlated with the patients' age or tumor size (Table 3 ). EGFR overexpression was significantly correlated with high IMP3 expression $(P<.0001)$.

\subsection{Prognostic analysis of IMP3 and EGFR}

Follow-up information was available in 112 cases, in which the follow-up time ranged from 0.4 to 368.5 months (median, 36.2 months). In total, as of the last follow-up, five patients (4\%) had died of the PT, 20 (18\%) were alive with disease, and $87(78 \%)$ showed no evidence of disease. Local recurrences were present in $24(21 \%)$ of 112 cases of all PTs, including $10(14 \%)$ of 70 benign PTs, $5(20 \%)$ of 25 borderline PTs, and 9/17 (53\%) malignant PTs. Metastasis occurred in 10/112 (9\%) cases, of which the primary tumor grade was benign in one $(1 \%)$ case, borderline in one $(4 \%)$ case and malignant in eight (47\%) cases. Among these 10 patients, five died of tumor and five were alive with metastatic disease. The first distant metastasis developed in lung $(\mathrm{n}=5)$, bone $(\mathrm{n}=4)$ and skin $(\mathrm{n}=1)$, although multi-organ metastases eventually occurred. All 10 of the metastatic cases had initially or eventually histologically malignant components; two cases of initially nonmalignant PT (one case each of benign and borderline PT) transformed into malignant PTs after several episodes of local recurrence, and eventually both patients died of metastatic tumor. Among these 10 cases, histological specimens of metastatic foci were available in six cases including five cases that were initially malignant PT and one case that was initially borderline PT. Overexpressions of IMP3 and EGFR were present in most of these six cases (6/6 and 5/6 cases, respectively). 


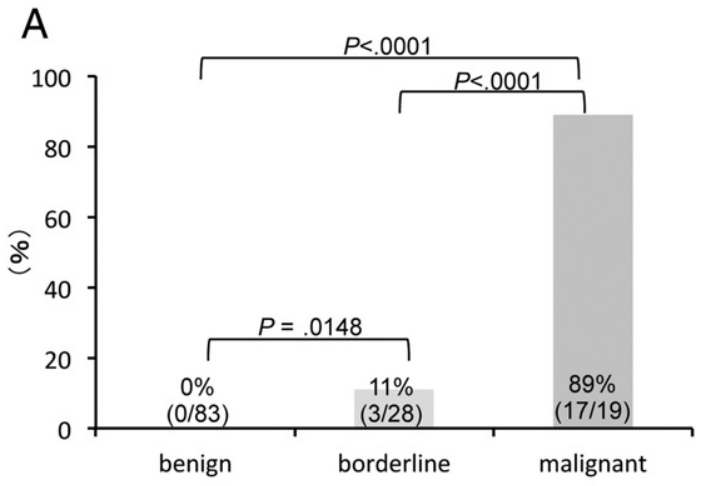

C

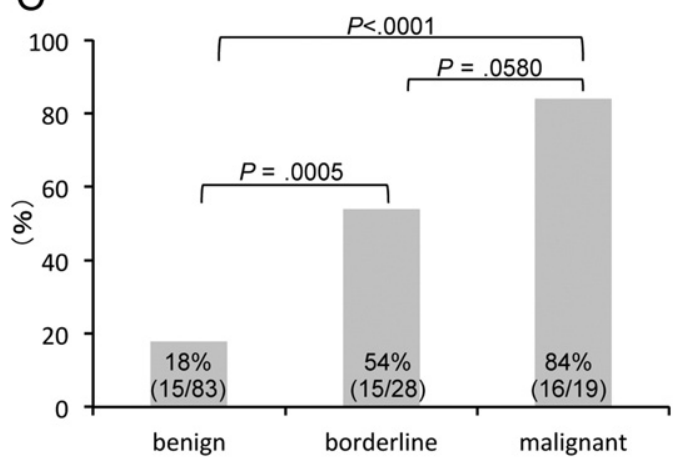

B

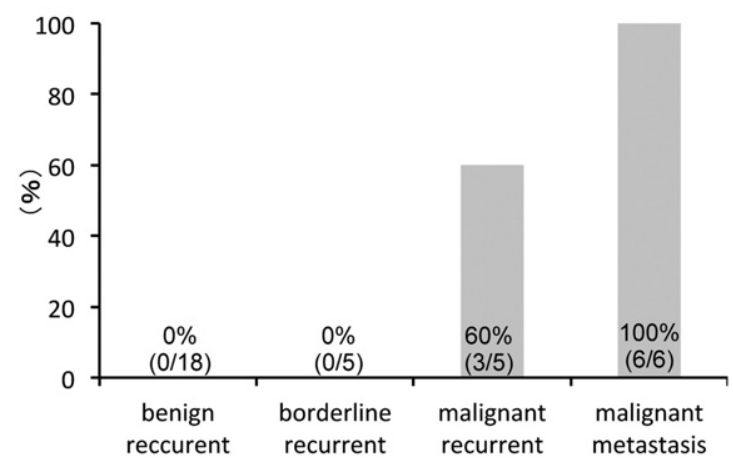

D

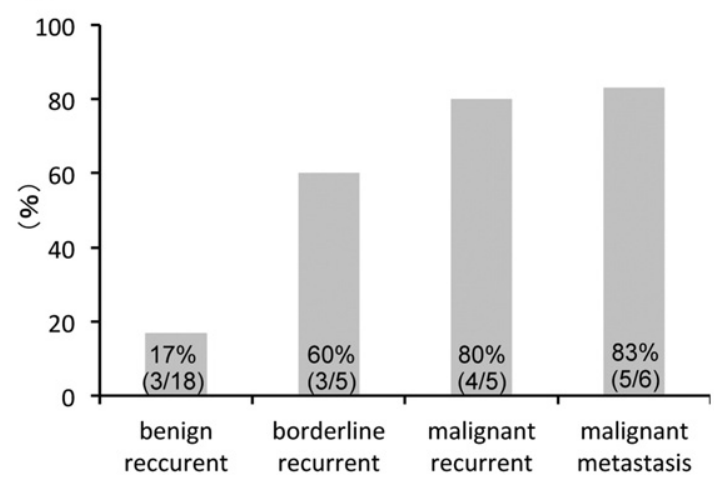

Fig. 2 The frequency of high IMP3 expression in each grade of primary PTs (A) and recurrent/metastatic PTs (B). The frequency of EGFR overexpression in each grade of primary PTs (C) and recurrent/metastatic PTs (D). High IMP3 expression (A) and EGFR overexpression (C) were each significantly more frequent in the higher-grade tumor among the primary tumors. The frequencies of high IMP3 expression (B) and EGFR overexpression (D) in the recurrent/metastatic tumors were essentially the same as those of the corresponding grades of primary tumors.

Table 3 The correlation between IMP3 and EGFR expression and clinicopathological parameters in primary PTs $(n=130)$

\begin{tabular}{|c|c|c|c|c|c|c|}
\hline \multirow[t]{2}{*}{ Factors } & \multicolumn{3}{|l|}{ IMP3 } & \multicolumn{3}{|l|}{ EGFR } \\
\hline & Low $(n=110)$ & $\operatorname{High}(n=20)$ & $P$ & Negative $(\mathrm{n}=84)$ & Positive $(n=46)$ & $P$ \\
\hline \multicolumn{7}{|l|}{ Age (years) } \\
\hline$\leq 45$ & 61 & 10 & \multirow[t]{2}{*}{.8077} & 45 & 26 & \multirow[t]{2}{*}{.8542} \\
\hline$>45$ & 49 & 10 & & 39 & 20 & \\
\hline \multicolumn{7}{|l|}{ Size $(\mathrm{cm})$} \\
\hline$\leq 5$ & 61 & 4 & \multirow[t]{2}{*}{.0064} & 45 & 20 & \multirow[t]{2}{*}{.3592} \\
\hline$>5$ & 49 & 16 & & 39 & 26 & \\
\hline \multicolumn{7}{|c|}{ Stromal cellularity } \\
\hline Score 1 & 73 & 0 & \multirow[t]{2}{*}{$<.0001$} & 60 & 13 & \multirow[t]{2}{*}{$<.0001$} \\
\hline Score 2,3 & 37 & 20 & & 24 & 33 & \\
\hline \multicolumn{7}{|c|}{ Nuclear atypia } \\
\hline Score 1 & 80 & 1 & \multirow[t]{2}{*}{$<.0001$} & 66 & 15 & \multirow[t]{2}{*}{$<.0001$} \\
\hline Score 2,3 & 30 & 19 & & 18 & 31 & \\
\hline \multicolumn{7}{|c|}{ Mitosis (/10 HPFs) } \\
\hline $0-4$ & 94 & 1 & \multirow[t]{2}{*}{$<.0001$} & 73 & 22 & \multirow[t]{2}{*}{$<.0001$} \\
\hline$\geq 5$ & 16 & 19 & & 11 & 24 & \\
\hline \multicolumn{7}{|l|}{ Ki-67 LI(\%) } \\
\hline$\leq 3 \%$ & 68 & 0 & \multirow[t]{2}{*}{$<.0001$} & 55 & 13 & \multirow[t]{2}{*}{$<.0001$} \\
\hline$>3 \%$ & 42 & 20 & & 29 & 33 & \\
\hline \multicolumn{7}{|l|}{ EGFR } \\
\hline Negative & 83 & 1 & \multirow[t]{2}{*}{$<.0001$} & - & - & - \\
\hline Positive & 27 & 19 & & - & - & - \\
\hline
\end{tabular}

Abbreviations: HPFs, high-power fields; LI, labeling index. 
We analyzed the prognostic values of the IMP3 overexpression, EGFR overexpression and other clinicopathological parameters. Histologically malignant tumors showed significantly shorter MFS and DFS $(P<.0001, P=.0010$, respectively) (Fig. 3A and D). High IMP3 expression was significantly associated with shorter periods of MFS $(P<.0001)$ and DFS $(P=.0007)$ (Fig. 3B and E). EGFR overexpression was also significantly associated with shorter periods of MFS $(P=.0023)$ and DFS $(P=.0211)$ (Fig. 3C and F). Histological grade, high IMP3 and EGFR expression were not each correlated with shorter OS $(P=.0991, P=$ .0601 and $P=.2337$, respectively).

In the univariate analysis, higher stromal cellularity score (score 2 or $3, P=.0011$ ), higher nuclear atypia score (score 2 or $3, P=.0004)$, higher mitoses ( 5 or more/10 HPFs, $P \leq$ $.0001)$, higher Ki-67 labeling index $(>3 \%, P=.0001)$, EGFR positive expression $(P=.0030)$ and high IMP3 expression $(P \leq .0001)$ were each significantly correlated with shorter MFS (Table 4).

In the multivariate analysis, high IMP3 expression was the only independent prognostic factor associated with shorter MFS (hazard ratio: $7.54, P=.0192$ ). We excluded mitosis and EGFR expression from the multivariate analysis, because there was a strong correlation between mitosis and $\mathrm{Ki}-67$ and that between EGFR expression and IMP3 expression (Table 3); these factors were thought to be confounding.

\section{Discussion}

The present study showed the prevalence and clinicopathologic significance of IMP3 expression in PTs. Very recently, Bellezza et al have shown that IMP3 expression was higher in malignant PTs $(56 \%$ of cases) than in borderline (15\%) and benign cases $(5 \%)$; however, the prognostic value of IMP3 overexpression in PT has been unclear [15]. In the current study, we found that the IMP3 expression was higher in malignant PTs compared to the fibroadenomas and benign to borderline PTs. In addition, the heterologous sarcomatous element and the malignant component in the tumors with a heterogeneous grade showed high expressions of IMP3, supporting our hypothesis that IMP3 overexpression may be related to histological progression. High IMP3 expression was also correlated with significantly worse prognosis in the present series. Notably, the high expression of IMP3 was an independent prognostic factor associated with shorter MFS in the multivariate analysis. In our series of PTs, all 10 of the cases with distant metastasis had initially or eventually histologically malignant components. In addition, the cause of death was distant metastasis in all five of the patients who died. These findings indicate that the correct pathological diagnosis to identify the histologically malignant component is very important for the clinical management of PT. As for IMP3, the majority of metastatic PTs showed high IMP3
A

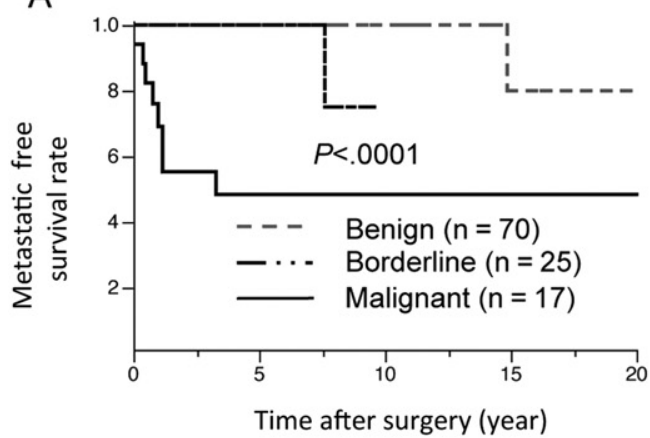

D

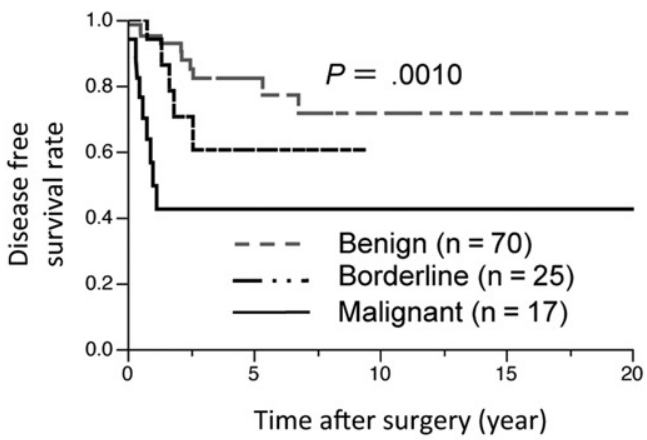

B

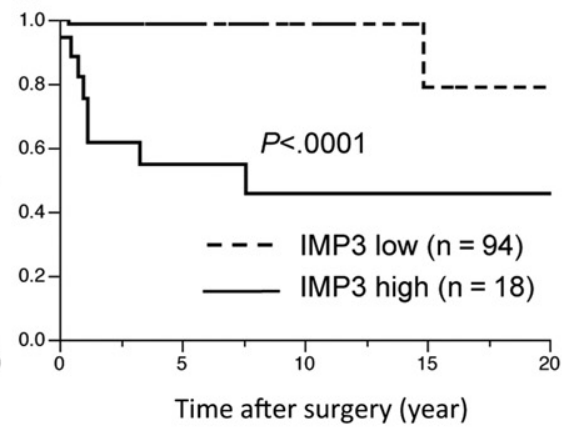

E

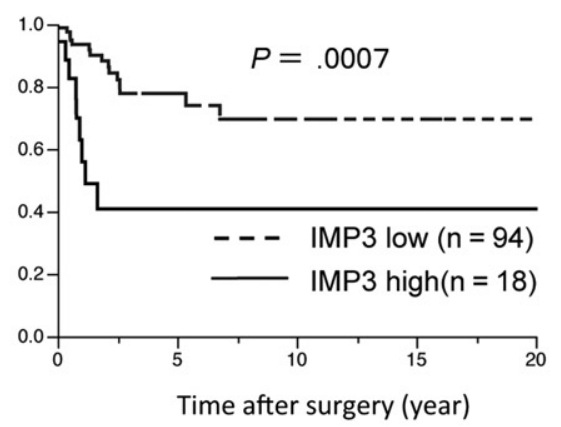

C

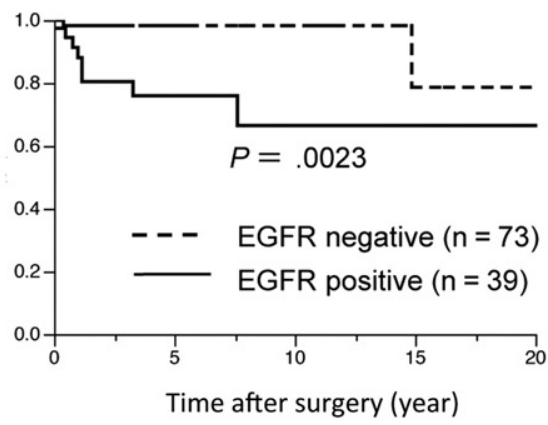

$\mathrm{F}$

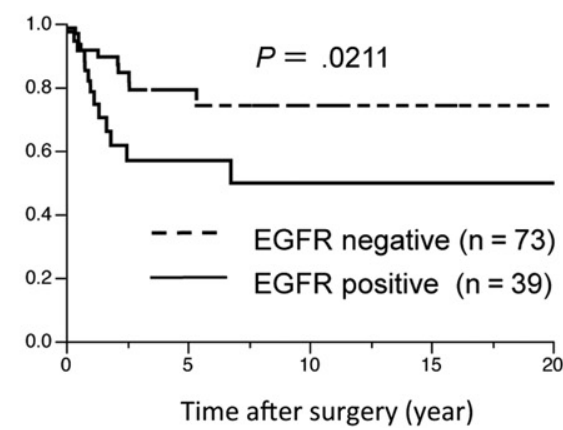

Fig. 3 Kaplan-Meier analyses for metastasis-free survival (A-C) and disease-free survival (D-F) by histological grade, IMP3 expression and EGFR expression. Among 110 cases of PTs, the histological grade (malignant PT) (A and D) high IMP3 expression (B and E) and EGFR overexpression $(\mathrm{C}$ and $\mathrm{F})$ were significantly associated with shorter survival times $(P<.0001, .0010,<.0001, .0007, .0023$, and .0211 , respectively). 
Table 4 Prognostic factors for metastasis-free survival in phyllodes tumors

\begin{tabular}{|c|c|c|c|c|}
\hline \multirow[t]{2}{*}{ Factor } & \multicolumn{2}{|l|}{ Univariate } & \multicolumn{2}{|l|}{ Multivariate } \\
\hline & Hazard ratio $(95 \% \mathrm{CI})$ & $P$ & Hazard ratio $(95 \% \mathrm{CI})$ & $P$ \\
\hline \multicolumn{5}{|l|}{ Age (years) } \\
\hline$\leq 45$ versus $>45$ & $0.85(0.22-2.97)$ & .7963 & not included & \\
\hline \multicolumn{5}{|l|}{ Size $(\mathrm{cm})$} \\
\hline$\leq 5$ versus $>5$ & $2.57(0.71-11.94)$ & .1533 & not included & \\
\hline \multicolumn{5}{|l|}{ Stromal cellularity } \\
\hline Score 1 versus score 2,3 & $13.09(2.45-241.38)$ & .0011 & $0.27(0.007-10.92)$ & .4590 \\
\hline \multicolumn{5}{|l|}{ Nuclear atypia } \\
\hline Score 1 versus score 2,3 & $15.83(2.97-292.01)$ & .0004 & $3.26(0.30-102.17)$ & .3926 \\
\hline \multicolumn{5}{|l|}{ Mitosis (/10 HPFs) } \\
\hline$\leq 4$ versus $>4$ & $20.13(3.77-371.41)$ & $<.0001$ & not included & \\
\hline \multicolumn{5}{|l|}{ Ki-67 LI(\%) } \\
\hline$\leq 3$ versus $>3$ & 12,389,769 (5.37-inf.) & .0001 & 3,326,005 (0-inf.) & .0659 \\
\hline \multicolumn{5}{|l|}{ EGFR } \\
\hline Negative versus positive & $7.68(1.92-50.90)$ & .0030 & not included & \\
\hline \multicolumn{5}{|l|}{ IMP3 } \\
\hline Low versus high expression & $18.46(4.56-123.17)$ & $<.0001$ & $7.54(1.33-141.30)$ & .0192 \\
\hline
\end{tabular}

expression. In this context, IMP3 may be an ancillary tool in identifying histologically malignant PTs and also PTs with high metastatic potential.

There are some reports showing that in PTs, recurrence-free survival is influenced by positive surgical margins [4,17]. Unfortunately, in our patient series, the surgical margin information was not available in several cases - the cases from two or three decades ago, in particular. Nevertheless, high IMP3 expression was also associated with shorter RFS, and it is thus possible that IMP3 overexpression contributes to local recurrence. However, the precise mechanism of any involvement of IMP3 in recurrence is not clear at present.

In benign to borderline PTs, stromal cellularity may be higher in the zone adjacent to the epithelial component, a phenomenon that is sometimes referred to as the periepithelial accentuation of stromal cellularity [2]. Sawhney et al showed that stromal mitotic activity in PT was significantly more likely to occur close to rather than remote from the epithelial component [18]. These observations support the hypothesis that stromal growth may be stimulated by the epithelial component $[18,19]$. In the present study, benign to borderline PT sometimes showed weak and focal IMP3 expression only at the periductal area. On the other hand, in parallel with stromal cell overgrowth, malignant PT showed diffuse and strong expressions of IMP3. These findings suggest that the overexpression of IMP3 in malignant PT may play a role in the growth of stromal cells in a manner independent of epithelial component.

Previous studies showed that EGFR expression was higher in advanced-grade PTs, indicating that EGFR overexpression may be involved in the progression of PTs $[13,14]$. In contrast, Yonemori et al have shown that EGFR expression was not correlated with recurrence-free or overall survival in $41 \mathrm{PT}$ cases
[20]. To the best of our knowledge, this is the first report showing that EGFR overexpression was correlated with worse metastasis-free and disease-free survival in PT. In addition, EGFR overexpression was significantly correlated with high IMP3 expression. According to an experimental study of TNBC, EGFR signaling pathway induced IMP3 overexpression, and IMP3 contributed to the migration and invasion of carcinoma cells [12]. In addition, estrogen receptor $\beta$ repressed the expression of EGFR, leading to the downregulation of IMP3 in TNBC cells. Similar to the relationship between EGFR and IMP3 in TNBC, overexpressed EGFR might contribute to the increased IMP3 expression especially in malignant PT, and IMP3 might play a role as an effector of EGFR-mediated tumor cell growth in PT. Although further research is needed to elucidate the relationship between EGFR and IMP3 at the molecular level, our results suggest that EGFR inhibition, leading IMP3 down regulation, might be a therapeutic strategy of malignant PT, like TNBC [12].

According to recent reports, the MED12 mutation is present in $60 \%$ to $70 \%$ of fibroadenomas and benign to borderline PTs $[21,22]$. In contrast, the MED12 mutation is present only in $20 \%$ to $40 \%$ of malignant phyllodes tumor. Considering this difference together with IMP3 expression status, some population of malignant PTs might have a distinctive molecular pathogenic mechanism which is different from that of fibroadenoma and benign to borderline PTs. However, further studies are warranted to elucidate this hypothesis and the relationship between MED12 and IMP3.

In conclusion, our findings suggest that high expressions of IMP3 and EGFR with a characteristic distribution (ie, a diffuse staining pattern) may be helpful for both identifying malignant PTs and predicting their prognoses. 


\section{Supplementary data}

Supplementary data to this article can be found online at http://dx.doi.org/10.1016/j.humpath.2016.04.007.

\section{References}

[1] Tse GM, Niu Y, Shi HJ. Phyllodes tumor of the breast: an update. Breast Cancer 2010;17:29-34.

[2] Tan PH, Tse G, Lee A, Simpson JF, Hanby AM. Fibroepithelial tumours. In: Lakhani SR, Ellis IO, Schnitt SJ, Tan PH, van de Vijver MJ, editors. World Health Organization classification of tumors. WHO classification of tumors of the breast. Lyon: IARC Press; 2012. p. 142-7.

[3] Moffat CJ, Pinder SE, Dixon AR, Elston CW, Blamey RW, Ellis IO. Phyllodes tumours of the breast: a clinicopathological review of thirtytwo cases. Histopathology 1995;27:205-18.

[4] Barth Jr RJ. Histologic features predict local recurrence after breast conserving therapy of phyllodes tumors. Breast Cancer Res Treat 1999;57: 291-5.

[5] Jara-Lazaro AR, Tan PH. Molecular pathogenesis of progression and recurrence in breast phyllodes tumors. Am J Transl Res 2009;1:23-34.

[6] Tse GM, Lee CS, Kung FY, et al. Hormonal receptors expression in epithelial cells of mammary phyllodes tumors correlates with pathologic grade of the tumor: a multicenter study of 143 cases. Am J Clin Pathol 2002;118:522-6.

[7] Nielsen J, Christiansen J, Lykke-Andersen J, Johnsen AH, Wewer UM, Nielsen FC. A family of insulin-like growth factor II mRNA-binding proteins represses translation in late development. Mol Cell Biol 1999;19: 1262-70.

[8] Liao B, Hu Y, Brewer G. RNA-binding protein insulin-like growth factor mRNA-binding protein 3 (IMP-3) promotes cell survival via insulin-like growth factor II signaling after ionizing radiation. J Biol Chem 2011;286: $31145-52$.

[9] Morimatsu K, Aishima S, Yamamoto H, et al. Insulin-like growth factorII messenger RNA-binding protein-3 is a valuable diagnostic and prognostic marker of intraductal papillary mucinous neoplasm. Hum PATHOL 2013;44:1714-21.
[10] Yamamoto H, Arakaki K, Morimatsu K, et al. Insulin-like growth factor II messenger RNA-binding protein 3 expression in gastrointestinal mesenchymal tumors. Hum PATHOL 2014;45:481-7.

[11] Cornejo K, Shi M, Jiang Z. Oncofetal protein IMP3: a useful diagnostic biomarker for leiomyosarcoma. Нuм PAтHоL 2012;43:1567-72.

[12] Samanta S, Sharma VM, Khan A, Mercurio AM. Regulation of IMP3 by EGFR signaling and repression by ER $\beta$ : implications for triple-negative breast cancer. Oncogene 2012;31:4689-97.

[13] Kersting C, Kuijper A, Schmidt H, et al. Amplifications of the epidermal growth factor receptor gene (egfr) are common in phyllodes tumors of the breast and are associated with tumor progression. Lab Investig 2006;86: 54-61.

[14] Tse GM, Lui PC, Vong JS, et al. Increased epidermal growth factor receptor (EGFR) expression in malignant mammary phyllodes tumors. Breast Cancer Res Treat 2009;114:441-8.

[15] Bellezza G, Prosperi E, Del Sordo R, Colella R, Rulli A, Sidoni A. IMP3 is strongly expressed in malignant phyllodes tumors of the breast: an immunohistochemical study. Int J Surg Pathol 2016;24:37-42.

[16] King RL, Pasha T, Roullet MR, Zhang PJ, Bagg A. IMP-3 is differentially expressed in normal and neoplastic lymphoid tissue. HuM PATHOL 2009;40:1699-705.

[17] Tan PH, Thike AA, Tan WJ, et al. Predicting clinical behaviour of breast phyllodes tumours: a nomogram based on histological criteria and surgical margins. J Clin Pathol 2012;65:69-76.

[18] Sawhney N, Garrahan N, Douglas-Jones AG, Williams ED. Epithelialstromal interactions in tumors. A morphologic study of fibroepithelial tumors of the breast. Cancer 1992;70:2115-20.

[19] Sawyer EJ, Hanby AM, Rowan AJ, et al. The Wnt pathway, epithelialstromal interactions, and malignant progression in phyllodes tumours. J Pathol 2002;196:437-44.

[20] Yonemori K, Hasegawa T, Shimizu C, et al. Correlation of p53 and MIB-1 expression with both the systemic recurrence and survival in cases of phyllodes tumors of the breast. Pathol Res Pract 2006;202: 705-12.

[21] Pfarr N, Kriegsmann M, Sinn P, et al. Distribution of MED12 mutations in fibroadenomas and phyllodes tumors of the breast-implications for tumor biology and pathological diagnosis. Genes Chromosom Cancer 2015;54:444-52.

[22] Ng CC, Tan J, Ong CK, et al. MED12 is frequently mutated in breast phyllodes tumours: a study of 112 cases. J Clin Pathol 2015;68: 685-91. 